\title{
« Faire sortir les déboutés ». Gestion, contrôle et expulsion dans les centres pour demandeurs d'asile en France
}

How "to make them leave": Administration, Control and Expulsion of Asylum Seekers in French Reception Centers

"Echar a los no admitidos". La gestión, el control y la expulsión en los centros para solicitantes de asilo en Francia

\section{Carolina Kobelinsky}

\section{(2) OpenEdition}

\section{Journals}

\section{Édition électronique}

URL : https://journals.openedition.org/conflits/15973

DOI : $10.4000 /$ conflits. 15973

ISSN : $1777-5345$

Éditeur :

CECLS - Centre d'études sur les conflits - Liberté et sécurité, L'Harmattan

\section{Édition imprimée}

Date de publication : 30 octobre 2008

Pagination : 113-130

ISBN : 978-2-296-06624-3

ISSN : 1157-996X

\section{Référence électronique}

Carolina Kobelinsky, « «Faire sortir les déboutés ». Gestion, contrôle et expulsion dans les centres pour demandeurs d'asile en France », Cultures \& Conflits [En ligne], 71 | automne 2008, mis en ligne le 03 février 2009, consulté le 21 septembre 2021. URL : http://journals.openedition.org/conflits/15973 ; DOI : https://doi.org/10.4000/conflits. 15973 


\title{
«Faire sortir les déboutés". Gestion, contrôle et expulsion dans les centres pour demandeurs d'asile en France
}

\section{Carolina KOBELINSKY}

Carolina Kobelinsky est doctorante en anthropologie à l'EHESS, rattachée à l'Institut de recherche interdisciplinaire sur les enjeux sociaux. Ses recherches portent sur la politique d'asile en France. Elle a récemment publié "The Moral Judgment of Asylum Seekers in French Reception Centers", Focus commentary, Anthropology News, mai 2008 (pp. 5-11).

\begin{abstract}
A rrêtée par des gendarmes dans un centre d'accueil de la région lyonnaise A en octobre 2007, une famille albanaise déboutée du droit d'asile fut envoyée dans un centre de rétention en vue de son expulsion. Malgré la mobilisation associative, le renvoi s'effectua quelques semaines plus tard. D'après la presse, les voisins assistèrent au déploiement en masse des forces de l'ordre et des pompiers dans le but d'empêcher que la famille s'échappe par la fenêtre. Déboutés par la Commission de recours des réfugiés, les membres de la famille étaient convoqués au Tribunal administratif après avoir demandé la suspension de la mesure d'éloignement ainsi qu'un réexamen du dossier. Les gendarmes se dirigèrent toutefois au Centre d'accueil pour demandeurs d'asile (CADA), forcèrent la porte de la chambre et conduisirent la famille - y compris les enfants - en rétention. Selon un journaliste de Libération, un responsable de l'association gestionnaire s'est défendu répétant: "C'est la loi, rien que la loi. On n'y peut rien ${ }^{1}{ }^{»}$.
\end{abstract}

Afin de contrôler les flux et de maîtriser les «indésirables ${ }^{2}$ ", les Etats européens mobilisent depuis plusieurs décennies un arsenal juridique et des

1. «Gendarmes et pompiers pour arrêter une famille », http://www.liberation.fr, mardi 16 octobre 2007.

2. Marrus M., The Unwanted: European Refugees in the Twentieth Century, New York, Oxford University Press, 1985. 
moyens matériels et policiers toujours plus importants. Les migrations illégales se sont constituées en un sujet prioritaire dans les débats à l'intérieur des pays communautaires ${ }^{3}$, les passeurs sont devenus les « criminels » responsables de l'augmentation des flux ${ }^{4}$, et la chasse aux « clandestins » est venue justifier la mise en place d'une politique sécuritaire. Dans ce contexte, la restriction progressive du droit d'asile fait partie des instruments de contrôle des frontières. Les « camps d'étrangers 5 » apparaissent comme un modèle dominant de gestion de la mise à l'écart des irréguliers, mais aussi des étrangers dont le statut juridique est précaire et soumis à évaluation, comme c'est le cas des demandeurs d'asile. Les pratiques déployées dans un centre de détention, un local de rétention, une zone d'attente ou un centre pour demandeurs d'asile, dessinent - malgré leurs différences - tout un ensemble d'espaces d'enfermement dans la circulation. Certainement plus douces par rapport aux institutions fermées où sont présentes les forces de l'ordre (que ce soit la police aux frontières ou la gendarmerie), les CADA participent de ce dispositif de mise à l'écart ${ }^{6}$. Le fait que l'on puisse aller chercher les déboutés du droit d'asile à leur "sortie » du centre, grâce au nouveau système de repérage, pour les conduire en rétention et les renvoyer - soit dans leur pays d'origine, soit dans un pays tiers qui accepterait de les recevoir -, invite à réinscrire cet espace de confinement et ces pratiques de contrôle dans un contexte de circulation à la marge où l'on trouve une constellation de lieux de passage, d'attente et d'enfermement.

A partir d'une enquête ethnographique menée durant vingt-cinq mois, entre 2004 et 2007 7, notamment dans deux CADA situés en banlieue d'une

3. Sassen S., Guests and Aliens, New York, The New Press, 1999.

4. Palidda S., «Passeurs, mediatori et intermediari », La Ricerca Folklorica, n44, Antropologia dei processi migratori, 2001.

5. Bernardot M., Camps d'étrangers, Bellecombre-en-Bauges, Editions du Croquant, 2008.

6. De nombreux travaux ont abordé la mise à l'écart des étrangers, voir notamment pour le contexte européen : Bernardot M., op. cit.; Bietlot M., «Du disciplinaire au sécuritaire : de la prison au centre fermé », Multitudes, n¹1, 2003, pp. 57-66; Caloz-Tschopp M.-C., Les Etrangers aux frontières de l'Europe et les spectres des camps, Paris, La Dispute, 2004 ; Courau H., Ethnologie de la forme-camp de Sangatte. De l'exception à la régulation, Paris, Editions des Archives contemporaines, 2007 ; Dal Lago A., Non-persone. L'esclusione dei migranti in una società globale, Milano, Saggi Universale Economica Feltrinelli, 2005 ; Le Cour Grandmaison O., Lhuilier P., Valluy J. (dirs.), Le Retour des camps ? Sangatte, Lampedussa, Guantanamo..., Paris, Autrement, 2007 ; Palidda S., Polizia postmoderna. Enografia del nouvo controllo sociale, Milano, Giangiacomo Feltrinelli Editore, 2000. Nous renvoyons également à Cultures $\mathcal{E}$ Conflits $\mathrm{n}^{\circ} 23$ : "Circuler, enfermer, éloigner : zones d'attente et centres de rétention aux frontières des démocraties occidentales »; $\mathrm{n}^{\circ} 49$ 《La mise à l'écart des étrangers. Les logiques du visa Schengen »; n50 « La mise à l'écart des étrangers. Les effets du visa Schengen »; n 57 "L'Europe des camps. La mise à l'écart des étrangers »; et au n ${ }^{\circ} 69$ de Politix « Etrangers : la mise à l'écart », avril 2005.

7. La démarche ethnographique choisie est notamment fondée sur l'observation (directe et participante) des interactions quotidiennes au sein du CADA ainsi que sur des entretiens semi-directifs avec des résidents et des travailleurs sociaux. Cette recherche a bénéficié des moyens et du cadre de réflexion qu'offrent le programme ASILES, financé par le ministère de la Recherche (projet ACI 3T.), dirigé par Michel Agier, et le programme «Les nouvelles frontières de la société française », financé par l'Agence nationale de la recherche et dirigé par Didier Fassin. 
grande agglomération, il s'agit ici d'étudier ce que « les sorties » des déboutés disent sur la gestion des demandeurs d'asile. L’expulsion des déboutés - dissimulée dans le terme fréquemment utilisé de "sorties »-semble incarner les logiques de rendement et de contrôle qui opèrent dans le traitement des demandeurs d'asile en CADA. Ces deux logiques convergentes apparaissent toutefois comme l'antithèse du travail social. "Faire sortir les déboutés 8 ", de manière plus ou moins forcée, est considérée comme une tâche pénible pour les intervenants des CADA et se pose de plus en plus comme un véritable dilemme pour les professionnels de cette institution à des fins assistantielles. L'article souhaite explorer le rôle ambigu des intervenants sociaux travaillant pour les associations gestionnaires de l'Etat lorsqu'il s'agit non plus seulement de contrôler mais aussi d'expulser les étrangers. Pour ce faire, après avoir brièvement décrit les structures d'accueil pour demandeurs d'asile, nous nous interrogerons sur le rôle des travailleurs sociaux au sein du dispositif et poserons le cadre juridico-administratif qui contraint les intervenants à gérer des flux. Il s'agira par la suite d'examiner les pratiques de contrôle dans le quotidien des CADA et, enfin, d'aborder la façon dont les intervenants pensent et gèrent effectivement les «sorties » des déboutés.

\section{Une structure d'accueil}

Financées par l'Etat et gérées par différentes associations loi 1901, les structures CADA hébergent des demandeurs d'asile de toutes origines pendant la durée de la procédure juridique d'évaluation de leur dossier. Elles voient le jour en 1991 9, deux mois après l'entrée en vigueur de la circulaire ministérielle supprimant le droit au travail des demandeurs d'asile ${ }^{10}$. Il s'opère alors une profonde transformation du dispositif, qui vient concrétiser dans le domaine de l'accueil le glissement sémantique qui s'opère au cours de la décennie précédente pour désigner les étrangers. Il y a trente ans, on ne parlait pas de «demandeurs d'asile », mais seulement de "réfugiés ». Au cours de la décennie 1980, le demandeur d'asile devient la catégorie administrative par excellence. Depuis la fin des années 1980, l'opinion publique (ré)installe la logique du « faux » avec, en vedette, la catégorie du «faux réfugié » ${ }^{11}$. Dix ans plus tard, il n'est plus seulement question de «faux réfugiés» mais également de «faux demandeurs

8. Cette expression est utilisée quotidennement par les travailleurs sociaux que nous avons rencontrés dans les CADA.

9. En application de la circulaire 91-22 du 19 décembre 1991, qui sera par la suite abrogée par la circulaire du 29 mars 2000.

10. Il s'agit de la circulaire du Premier ministre NOR/PRM/X/91/00102/60/D du 26 septembre 1991, dite circulaire Cresson.

11. Le discours qui oppose des «vrais» et des «faux réfugiés » peut se retrouver à la fin des années 1950, lorsqu'une présomption négative pèse sur les exilés yougoslaves perçus, en raison de leur faible niveau de qualification et de diplômes, comme une « immigration de main-d'œuvre », à la différence de l'immigration hongroise, davantage composée d'étudiants et d'intellectuels diplômés, et perçue comme des «vrais réfugiés". Spire A., Etrangers à la carte. L'administration de l'immigration en France (1945-1975), Paris, Grasset, 2005, p. 228. 
d'asile $12 »$. Il existe donc depuis le début des années 1990 une distinction - et une scission - entre demandeurs d'asile et réfugiés, accueillis par des structures distinctes. Les Centres provisoires d'hébergement $(\mathrm{CPH})$, qui prenaient jusqu'alors en charge des personnes reconnues comme réfugiées et des demandeurs d'asile, s'occupent désormais exclusivement des réfugiés, et les nouveaux CADA sont institués pour recevoir des requérants en cours de procédure.

Le dispositif national d'accueil qui pilote l'ensemble des structures ayant pour objet l'hébergement des demandeurs d'asile et des réfugiés ainsi que l'insertion sociale et professionnelle des statutaires ${ }^{13}$ est longtemps géré par l'association France Terre d'asile (FTDA). Depuis janvier 2004, la coordination revient à l'Office des migrations internationales (OMI), organisme public relevant du ministère chargé du travail, devenu en 2005 l'Agence nationale de l'accueil des étrangers et des migrations (ANAEM) ${ }^{14}$. Les CADA offrent avant tout un logement provisoire aux demandeurs d'asile accueillis par le dispositif. L'hébergement est soit éclaté (c'est-à-dire dans des appartements situés en dehors du bâtiment administratif du CADA, avec, par conséquent, un éparpillement des « résidents $15 »$ dans toute une ville ou une région), soit collectif (les résidents sont regroupés dans un bâtiment de type foyer). L'enquête de terrain sur laquelle s'appuie cet article a été effectuée dans des institutions d'hébergement collectif, plus nombreuses, qui assurent un suivi sanitaire, une aide pour le dossier juridique (c'est-à-dire pour la construction du récit de persécution que le demandeur doit présenter à l'OFPRA et, en cas de recours, à la Cour nationale du droit d'asile), la scolarisation des enfants de moins de 16 ans ainsi qu'un accompagnement social.

Tout comme le statut de réfugié, le CADA constitue une ressource rare à laquelle la plupart des demandeurs d'asile n'ont pas accès. Antoine Decourcelle et Stéphane Julinet 16 constataient, il y a huit ans de cela, la saturation de ces

12. Si la prééminence terminologique penche désormais pour les « déboutés ", c'est moins à cause des médias que parce que, de fait, le droit d'asile est devenu une machine à produire des rejetés. Si, en 1973, l'Office français de protections des réfugiés et des apatrides (OFPRA) accordait le statut à $85 \%$ des requérants, en moins de vingt ans, on assiste à un renversement des proportions et seuls $15 \%$ des demandeurs obtiennent le statut en 1990 (voir Legoux L., La Crise de l'asile politique en France, Paris, CEPED, p. 138). En 2007, l'OFPRA a rejeté $88,4 \%$ des demandes, le taux global d'admission (somme des décisions d'accord de l'OFPRA et des décisions d'annulation de la Commission des recours) étant de $29,9 \%$ (voir : http://www.ofpra.gouv.fr, Rapport annuel de l'OFPRA, 2007, p. 23).

13. Le dispositif financé par l'aide sociale est actuellement composé de trois structures: les $\mathrm{CADA}$, les $\mathrm{CPH}$ et les Centres de transit, qui hébergent des demandeurs d'asile en attente d'une place en CADA. Il existe également depuis 2000 un dispositif d'accueil d'urgence spécifique pour demandeurs d'asile (AUDA), géré, à la demande de la Direction de la population et des migrations, par la SONACOTRA, société d'économie mixte, devenue Adoma en 2007. De capacité variable selon les années, ces structures offrent un hébergement et un accompagnement social et juridique pendant la période d'hiver.

14. Cette agence fusionne les compétences et les moyens de l'OMI et du Service social d'aide aux émigrants (SSAE).

15. C'est ainsi que les demandeurs d'asile sont catégorisés à leur entrée en CADA.

16. Decourcelle A., Julinet S., Que reste-t-il du droit d'asile ?, Paris, L’Esprit frappeur, 2000. 
structures. Malgré l'augmentation progressive du nombre de places, les CADA n'arrivent toujours pas à accueillir plus d'un quart de la totalité des requérants ${ }^{17}$. Ainsi, la majeure partie des demandeurs n'accède pas au dispositif d'accueil ; ils ne pouvaient compter, jusqu'en novembre 2006, que sur une aide financière durant 12 mois, alors même que la procédure d'asile excédait souvent ce délai. Désormais, cette aide est remplacée par une nouvelle allocation versée pendant toute la durée de la procédure ${ }^{18}$. Les résidents du CADA ont alors le double «privilège » d'être assistés pendant une période plus longue et de bénéficier d'une aide technique pour monter leur dossier. Cela n'est pas négligeable quant au taux d'obtention du statut considérablement plus élevés pour les requérants accueillis en CADA. L'« écrémage » qui s'effectue au moment du choix des candidats pour l'attribution des places du dispositif peut également constituer un facteur explicatif à ces succès. Les places en CADA sont à la fois un objet de convoitise pour les demandeurs d'asile et un enjeu (politique, économique) de mobilisation pour leurs défenseurs. Car, comme le souligne Jérôme Valluy ${ }^{19}$, l'importance financière de ces structures pour les associations qui les gèrent est loin d'être négligeable, le prix de la journée étant facturé à $24,82 €$ par personne. Les associations gestionnaires sont devenues très dépendantes de ces financements pour payer leurs salariés, dont le nombre a brutalement augmenté depuis la fin des années 1990, ce qui est à mettre en relation directe avec l'augmentation du nombre de places d'accueil en CADA.

Les demandeurs d'asile sont admis en CADA suite à la décision d'une commission d'admission selon des «critères d'urgence sociale ». La Commission nationale d'admission (CNA), animée jusqu'au 1er janvier 2004 par la FTDA, avec le soutien du Service social d'aide aux émigrants et présidée par un représentant de la Direction des populations et des migrations, s'occupait de sélectionner les bénéficiaires de cet accueil et de les répartir dans les différents départements disposant de centres d'accueil. Depuis janvier 2004, le secrétariat de la CNA est assuré par l'ANAEM (anciennement OMI). On assiste désormais à une déconcentration du dispositif d'admission, partageant le travail entre la CNA et les Commissions locales d'admission (CLA), ces dernières étant placées sous la direction des Directions départementales des Affaires sanitaires et sociales (DDASS) ${ }^{20}$. Les membres de ces commissions sont des fonctionnaires

17. On passe de 3588 places en 1998 à environ 20000 dix ans plus tard. Voir Van Erkelens A., "Comment est géré un Centre d'accueil de demandeurs d'asile lorsqu'il donne lieu à une convention entre l'Etat et une association ou une entreprise ? ", Paris, TERRA-Editions, coll. «Synthèses », $2005:$ http://terra.rezo.net/article442.html

18. Depuis le 16 novembre 2006, l'allocation d'insertion versée par les ASSEDIC est remplacée par l'allocation temporaire d'attente (ATA), décret n²006-1380 du 13 novembre 2006.

19. Valluy J., "L'accueil étatisé des demandeurs d'asile : de l'enrôlement dans les politiques publiques à l'affaiblissement des mobilisations de soutien aux exilés ", Paris, TERRA-Editions, coll. « Esquisses », 2007 (http://terra.rezo.net/article556.html) ; Valluy J., Sociologie politique de l'accueil et $d u$ rejet des exilés, thèse d'Habilitation à diriger des recherches, université Robert-Schuman, Strasbourg-II, 2008. Version en ligne : http://terra.rezo.net/article794.html, tome 2, p. 220.

20. Pour une étude du fonctionnement des commissions d'admission, voir Franguiadakis S., 
et des directeurs d'associations gestionnaires des CADA. Les candidatures peuvent être présentées par toute association contactée par un demandeur d'asile. Les priorités sociales d'accès en CADA sont les suivantes : primo-arrivants (les demandeurs qui arrivent pour la première fois en France); familles avec enfants en bas âge ; jeunes majeurs isolés ; sur avis médical motivé, demandeurs d'asile ayant des problèmes de santé, mais ne nécessitant pas un accueil médicalisé ; femmes seules; demandeurs en réexamen n'ayant pas été hébergés ${ }^{21}$.

D’après un responsable de CADA, les CLA sélectionnent les résidents qui occuperont les places disponibles dans la région "selon les critères de vulnérabilité mentionnés dans la circulaire ». Tout le monde est une victime, «ce pauvre monde qui frappe partout à la porte pour demander protection " 22, mais il faut sélectionner les plus vulnérables d'entre eux ${ }^{23}$, car le nombre de candidats éligibles sur chacun des critères ci-dessus dépasse très largement les capacités d'accueil, ce qui contraint à faire un sélection à l'intérieur même des catégories éligibles. C'est dans ces espaces d'indéfinition des règles formelles ce «flou du droit » correspondant aux marges d'appréciation dans l'application des critères - que peut se greffer une préoccupation d'un tout autre ordre : la probabilité de réussite d'un requérant lors du futur examen de sa demande d'asile ${ }^{24}$ par l'Office français de protection des réfugiés et apatrides (OFPRA) et la Cour nationale du droit d'asile (CNDA) ${ }^{25}$. Les seuls immigrés qu'on laisse entrer en nombre très limité sont ainsi de plus en plus sélectionnés via différents mécanismes et filtres, afin de s'assurer, avant même l'examen de leurs demandes, qu'il s'agit bien de « bons » candidats à l'asile.

\section{Gérer des flux}

Le travail mené par les intervenants sociaux auprès des demandeurs d'asile au sein du CADA est inscrit dans un cadre procédural et référé à des missions institutionnelles permettant d'offrir un accompagnement qui fonctionne comme une intermédiation entre les résidents et une offre de biens sociaux, comme l'attribution de l'allocation globale mensuelle, les formations ou le

Jaillardon E., Belkis D., Bernigaud S., En quête d'asile. Aide associative et accès au $(x)$ droit(s), Paris, LGDJ, 2004.

21. Conformément à la circulaire DPM/CI 3 n99-399 du 8 juillet 1999.

22 . Entretien avec le directeur général de France Terre d'asile, « Réfugiés : on confond protection et ordre public », http://www.humanite.fr, 22 octobre 2003.

23. Comme le note Veena Das, être vulnérable n'est pas la même chose qu'être une victime. Parlant d'une femme veuve dans l'univers de parenté propre à l'ethos de la caste aisée Hindou, l'anthropologue explique qu'elle était déjà vulnérable (par sa condition de veuve) avant de devenir une victime de la partition de l'Inde en 1947. Voir Das V., “The act of witnessing. Violence, poisonous knowledge, and subjectivity”, in Das V., Kleinman A., Ramphele M., Reynolds P., Violence and Subjectivity, University of California Press, 2000, p. 209.

24. Valluy J., «L'accueil étatisé des demandeurs d'asile : de l'enrôlement dans les politiques publiques à l'affaiblissement des mobilisations de soutien aux exilés », op. cit., p. 6.

25. La CNDA remplace l'ancienne Commission des recours des réfugiés par l'article 29 de la loi du 20 novembre 2007. 
logement (pour ceux qui ont obtenu le statut). Les activités sociales proposées dans le cadre de l'accueil sont les suivantes : accompagnement social et médical, accompagnement juridique, animation, cours de FLE (Français langue étrangère), suivi scolaire. Selon la taille et le budget du CADA, les intervenants sociaux peuvent prendre en charge une ou plusieurs fonctions ${ }^{26}$.

Si le CADA est conçu comme un espace du social, les intervenants conviennent que l'accueil est de plus en plus soumis à une « logique du chiffre » de type managériale et axé sur la circulation (sous contrainte) des flux, ce qui est contraire aux fondements mêmes de leur activité :

Lors d'un déjeuner au restaurant, table à quatre, une travailleuse sociale se confiait :

«Je suis pessimiste, mais je sens que je ne fais plus de l'accompagnement social, que tout ce que je fais relève de l'administratif. Je ne suis plus dans le social [avant] je sentais que je participais à une réflexion. Maintenant, il n'y a plus de réflexion, il n'y a plus de social. On travaille pour entrer dans le cadre du règlement, pour faire du chiffre et c'est tout. »

(Notes de terrain, 17 février 2004)

Dans ce fragment se dessinent deux conceptions du rôle professionnel des intervenants : le référent social comme un administratif qui fait du chiffre et le médiateur entre la société et les «désaffiliés » dont le travail impliquerait un certain engagement. Dans le premier cas, le professionnel s'éloigne du social pour se soumettre à la rationalité économique du management, tandis que dans le deuxième il revendique son rôle ("classique ») de réparateur des souffrances infligées par la société. Une troisième image rattache le travail social à un rôle répressif :

26. Quelle que soit leur fonction, les travailleurs sociaux peuvent tous être considérés à la manière des street-level bureaucrats (Lipsky M., Street-Level Bureaucracy. Dilemmas of the Individual in Public Services, New York, Russel Sage Foundation, 1980) dont le travail implique un contact direct avec les personnes (non bureaucrates), dans ce cas, les demandeurs d'asile. Il faut pourtant signaler qu'à cette proximité, vient s'ajouter au CADA la dimension temporelle, toute particulière, des rapports qui s'établissent, qui instaurent une relation quotidienne et dans la durée. Les intervenants sociaux côtoient les résidents dans leur lieu de vie, ils se croisent au quotidien, parfois à plusieurs reprises dans une même journée. Dans ce cadre, il est difficile pour une bonne partie des travailleurs sociaux de rester émotionnellement détachés ou de maintenir une distance avec les résidents. A la différence du bureaucrate idéal-typique wébérien, qui remplit sa fonction formellement de manière égale pour " tout le monde ", au CADA, les rapports sont personnels et traversés par des sentiments et des valeurs. Voir Dubois V., $L a$ Vie au guichet. Relation administrative et traitement de la misère, Paris, Economica, 1999; Herzfeld M., The Social Production of Indifference. Exploring the Symbolic Roots of Western Bureaucracy, New York, Berg Publishers, 1992. La circulaire récente du 24 juillet 2008 $\left(n^{\circ}\right.$ NOR IMIA0800035 C) ajoute à ces missions celle de la gestion des sorties du centre 
«Je pense que dans la politique actuelle, le travail social est dans le contrôle. On est les moyens humains et on gère des moyens financiers dont l'objectif, c'est le contrôle. On est des comptables, on est des flics. La marge de manœuvre des travailleurs sociaux est de plus en plus réduite. De plus en plus, on va être contraint à la contrainte et en même temps, c'est du saupoudrage. La politique sociale est au contrôle et pas à l'entraide. Ça pervertit les travailleurs sociaux. »

(Entretien avec Estelle 27, intervenante dans un CADA, 30 novembre 2007).

Les « sorties » des déboutés du CADA incarnent la logique du chiffre qui transforme les travailleurs sociaux en " comptables ", à laquelle vient se greffer une autre politique, qui met le contrôle des populations au cœur du dispositif, faisant des intervenants de véritables «flics » ${ }^{28}$. Elles sont toutes deux perçues par une majeure partie des intervenants rencontrés en CADA comme l'antinomie « du social ». Lors des « sorties », les professionnels sont confrontés à une image d'eux-mêmes (de leur rôle) qu'ils ont du mal à accepter, voire qu'ils refusent. Ils se trouvent pris entre des exigences contradictoires, tiraillés, pour emprunter les termes d'Alain Morice, entre "leur idéal du bien public » et « le respect de la consigne » 29 . Les «sorties » deviennent ainsi un véritable dilemme pour les travailleurs sociaux, qui se voient clairement, à partir des dernières réformes législatives, comme les agents d'un dispositif visant à l'expulsion des étrangers.

L'Etat fait pression pour augmenter le taux de rotation en CADA, calculé comme le rapport du nombre d'entrées au nombre de places du centre, estimant que la fluidité du dispositif implique une meilleure rentabilité. Si le statut de réfugié leur est reconnu, les résidents disposent, en théorie, d'un délai de trois mois (renouvelable une fois) pour quitter le CADA. Pendant ce temps, soit ils trouvent ce qu'on appelle une " solution individuelle », c'est-àdire qu'ils ne font pas appel à l'aide sociale de l'Etat, soit ils sollicitent une orientation en CPH. Si leur demande n'aboutit pas, les résidents doivent quitter les locaux au bout d'un mois. Dès le 1 er janvier 2006, les directeurs de cabinet des ministres de l'Intérieur (Nicolas Sarkozy) et de l'Emploi (JeanLouis Borloo) demandent aux préfets de «veiller à améliorer le taux de rotation en $C A D A »$. Une note d'instruction indique aux préfets qu'il leur

27. Afin de respecter l'anonymat de nos interlocuteurs, tous les noms ont été changés.

28. L'association entre travail social et contrôle des populations considérées comme déviantes se retrouve déjà au début des années 1970. Voir notamment le numéro de la revue Esprit, "Pourquoi le travail social ?", avril-mai 1972, dans lequel la critique sociologique porte sur l'effet normalisateur du travail social, inscrit au registre des institutions disciplinaires. Cette approche apparait comme l'envers de la version positive qui fait du travail social « classique » une entreprise de réhabilitation des individus en vue de leur intégration dans la société.

29. Morice A., «Du SSAE à l'ANAEM : une liquidation annoncée », Plein droit, nº72, mars 2007, pp. 8-13. 
"appartient d'organiser sans délai, en lien avec le gestionnaire, la sortie $d u$ $C A D A$ des personnes bébergées qui n'ont désormais plus aucun droit à s'y maintenir en intervenant pour assurer l'éloignement effectif de ces déboutés encore hébergés en CADA 30 ». C'est d'ailleurs ce que d'autres textes viennent renforcer par la suite. La loi du 24 juillet 2006 relative à l'immigration et à l'intégration, dite loi Sarkozy, crée un fichier informatique des demandeurs d'asile et de suivi des mouvements d'entrées et sorties de CADA et oblige les gestionnaires des centres à transmettre à l'autorité de tutelle l'ensemble des données concernant les résidents. Cette base de données fournit aux préfets des informations sur la localisation des déboutés récents, donc susceptibles d'être arrêtés en vue de leur expulsion. Le décret du 23 mars 2007 poursuit dans le même sens, mettant en place le dispositif d'assignation dans des CADA et introduisant l'obligation de transmettre l'information aux préfets dans un délai d'un mois. Les demandeurs d'asile ne peuvent désormais y être admis et les quitter qu'avec l'autorisation du préfet, qui peut les suivre grâce au nouveau fichier informatique ${ }^{31}$. La circulaire du 3 mai 2007 précise, quant à elle, qu'ayant épuisé les droits au maintien en CADA, le préfet fera procéder à l'interpellation du débouté "soit dans les espaces collectifs $d u C A D A$, avec l'accord du gestionnaire, soit sur la voie publique».

Tous ces textes renforcent clairement l'association entre le dispositif assistantiel et les technologies de contrôle. Ils induisent une redéfinition du rôle des intervenants du CADA qui doivent mettre en œuvre ces dispositions. Or, l'accueil des demandeurs d'asile est marqué par des pratiques de contrôle au quotidien au-delà des « sorties ».

\section{Contrôler les résidents}

Sans droit au travail, les demandeurs d'asile deviennent économiquement complètement dépendants de l'aide sociale de l'Etat ${ }^{32}$. L'allocation offerte par le CADA recouvre la prise en charge de l'hébergement, plus une somme d'ar-

30. Note d'instruction interministérielle DPM/ACI3 n²006-31 du 20 janvier 2006 relative aux procédures d'admission et aux délais de séjour dans le dispositif national d'accueil des demandeurs d'asile adressée aux préfets et au directeur de l'ANAEM.

31. Si les demandeurs d'asile refusent d'entrer en CADA, l'allocation temporaire d'attente leur est retirée.

32. Par transposition de la directive européenne " accueil ", le décret du 23 août 2005 (n²0051051) prévoit que les demandeurs d'asile accèdent au marché du travail si l'OFPRA n'a pas statué sur la demande au bout d'un an. Dans ce cas, le demandeur d'asile est soumis aux règles de droit commun applicables aux travailleurs étrangers pour la délivrance d'une autorisation provisoire de travail. L'accès au marché de l'emploi étant prioritairement réservé aux nationaux et aux étrangers en situation régulière, il ne sera autorisé à occuper un poste que si, dans la branche d'activité et la zone géographique considérées, le nombre de demandes d'emploi n'est pas supérieur au nombre d'offres. L'obtention de cette autorisation est, de fait, exceptionnelle et dans la plupart des départements, la politique administrative est de refuser systématiquement le droit de travailler aux demandeurs d'asile, quelle que soit la situation de l'emploi. Voir CNCDH, Les Conditions d'exercice du droit d'asile en France, Paris, La Documentation fran- 
gent liquide pour subvenir aux besoins alimentaires. L'allocation globale mensuelle pour un couple s'élève à 267 euros, à 318 euros pour une famille de trois membres, et à 405 euros pour une famille de quatre personnes ${ }^{33}$. A cela, il faut ajouter les titres de transport qui leur sont donnés pour se rendre à l'OFPRA ou à la CNDA, ainsi qu'aux rendez-vous médicaux. Des «petites aides » peuvent éventuellement être attribuées de manière discrétionnaire à certains résidents dont l'histoire émeut et provoque la compassion des professionnels du centre ${ }^{34}$. En outre, les revenus d'un travail « au noir » viennent parfois compléter l'aide sociale qui s'avère insuffisante, permettant également de contourner ou d'estomper la mise en attente ${ }^{35}$.

A la dépendance économique s'ajoute le contrôle de l'activité. Quel que soit son mode d'organisation (plus grand ou plus petit, avec des appartements ou des chambres), le CADA est une institution partiellement fermée. Bien qu'on puisse établir des parallèles avec les situations décrites par Erving Goffman ${ }^{36}$, il ne s'agit pas à proprement parler d'une institution totale dans le sens où la circulation des résidents est certainement contrôlée mais non pas interdite : les demandeurs d'asile peuvent entrer et sortir de l'établissement. Cependant, selon le règlement intérieur des CADA, que les résidents doivent signer à leur arriver avec le contrat de séjour :

« le demandeur d'asile qui souhaite s'absenter plus d'un jour doit en informer l'équipe du centre. Toute absence de plus de cinq jours doit être autorisée par le responsable du centre. A défaut, elle sera considérée comme un départ volontaire, justifiant la fermeture de la chambre, la mise sous consigne des bagages et la fin de la prise en charge au titre de l'aide sociale ».

L'entrée de personnes inconnues du personnel est également problématique, notamment dans l'un des CADA où nous avons effectué notre terrain, où le contrôle est exercé de manière plus formelle dès lors que les responsables de l'institution soupçonnent certains résidents de permettre à des compatriotes

çaise, 2006, p. 147. Vaut-il la peine de mentionner que nous n’avons jamais rencontré un demandeur d'asile en CADA qui ait demandé une telle autorisation ?

33. Ce barème est établi selon la circulaire du ministère de l'Emploi et de la Solidarité MES/DPM n²000-170 du 29 mars 2000 relative aux missions des CADA et la note du ministère de l'Emploi et de la Solidarité du 16 janvier 2002 relative à la mise en place de l'euro.

34. Kobelinsky C., "Le jugement quotidien des demandeurs d'asile », Paris, TERRA-Editions, coll. «Esquisses », fevrier 2007 : http://terra.rezo.net/article559.html

35. Le travail permet de contourner les effets lourds du CADA : le contrôle, le confinement, etc. Il assure une entrée d'argent qui vient s'ajouter à l'aide de l'Etat et qui s'avère capitale pour couvrir les frais d'avocat. Cela permet d'ouvrir une voie de socialisation, de récupérer une certaine estime de soi et cela remplit l'attente - « activité » par excellence de ceux qui demandent l'asile. Le temps de travail occupe, il permet de cadrer le quotidien à partir d'une temporalité singulière associée au travail (dates, horaires, délais particuliers).

36. Goffman E., Asiles. Etudes sur la condition sociale des malades mentaux et autres reclus, Paris, Editions de Minuit, 1968. 
de s'introduire « clandestinement »-terme employé par un intervenant - dans les locaux pour dormir dans les parties communes. Dans ce CADA, les nouveaux arrivants reçoivent une «feuille de rendez-vous » que les référents sociaux remplissent minutieusement en indiquant les réunions des familles avec les différents services du Centre. Dans le second CADA où nous avons enquêté, la «feuille de rendez-vous » n'est pas institutionnalisée comme telle mais, de fait, les intervenants organisent les activités des nouveaux résidents pendant les premières semaines du séjour. Il s'agit surtout de contraintes bureaucratiques mais également d'activités récréatives et d'animation organisées par l'équipe du CADA. Le courrier postal des demandeurs d'asile est reçu par l'équipe du centre. Les résidents doivent aller le chercher aux bureaux des intervenants et leurs lettres officielles doivent être ouvertes devant un intervenant qui en fait une photocopie. Par ailleurs, les chambres sont systématiquement visitées par deux intervenants qui font l'état des lieux communs, discutent avec chaque résident sur les rapports avec les voisins. Pendant ces "visites d'appartement ", dont le but est de "contrôler qu'il n'y ait pas des dérives ", les intervenants ont leur mot à dire concernant le rangement, le ménage et les habitudes des résidents. Plusieurs demandeurs d'asile rencontrés nous ont fait part de leur sentiment d'être "traités comme des enfants à l'école». Certains commentaires des intervenants, énoncés dans des cadres plus informels, visent également à exercer un contrôle sur les activités quotidiennes des résidents.

Un intervenant, voyant de loin une demandeuse d'asile qui vient chercher son courrier, la salue en lui disant, sur un mode de plaisanterie :

«Ah, vous voilà, ça fait longtemps qu'on ne vous a pas vue dans le cours de français... [sourires de la résidente] Je me demande ce que vous faites, on ne vous voit pas beaucoup de ce temps-ci... Comment allez-vous ? Très occupée, $\mathrm{j}$ 'imagine... » (notes de terrain, CADA, 13 juin 2005)

Ce mode badin voire ironique est couramment employé par les intervenants sociaux lorsqu'ils font des commentaires à propos des horaires décalés (lorsque les résidents arrivent en retard, pour récupérer les jetons pour la laverie ou le courrier ; lorsqu'on suspecte qu'ils passent leur journée à dormir) ou de l'absentéisme des résidents lors des réunions du CADA.

Toutes ces pratiques sont vécues par les demandeurs d'asile que nous avons rencontrés comme l'expérience d'une infantilisation par l'équipe sociale du CADA, qui contrôle leurs activités et leur délivre l'allocation, dont le montant pourrait faire l'objet de ponctions si jamais ils contrevenaient au règlement intérieur du foyer. Bien que cela n'ait jamais eu lieu de manière effective - pendant le temps de notre enquête, tout du moins - cette clause fonctionne à la manière d'une menace de coercition qui peut éventuellement être 
évoquée par les salariés afin de veiller au respect des normes. Le CADA crée ainsi un «effet de dépendance accentué par le manque de marge de manouvre qui leur est offert dans ces centres 37 ». Des phrases telles que « ici on est comme à l'école », "on devient de petits enfants ", "on doit obéir » apparaissent dans la plupart des entretiens et des conversations avec les résidents.

Suivant l'exemple de son père, Simone Oyono s'intéressa très tôt à la vie politique de son pays et adhéra à l'Union nationale camerounaise. Après avoir passé quelques temps en prison, son père mourut et Simone s'engagea plus activement dans le parti. Elle fut arrêtée plusieurs fois, sa maison fouillée... Elle fut enfermée pendant quelques semaines et, lorsqu'elle fut libérée, elle prît la décision de partir et de rejoindre sa fille unique. Après trois semaines de voyage, cachée dans un bateau, Simone arriva en France. Elle réussit à monter jusqu'à Paris où elle retrouva sa fille de 14 ans, qu'elle avait fait venir chez des connaissances quelque temps avant sa fuite du Cameroun. Elles étaient au foyer depuis deux mois lorsque nous avons fait leur connaissance. Tout en reconnaissant le «soulagement» qu'elle a ressenti lorsqu'elle est arrivée en CADA où ses besoins fondamentaux, ainsi que ceux de sa fille, seraient garantis pendant quelque temps et où elle pourrait « reprendre des forces » 38 , Simone me fit part de son sentiment d'enfermement.

«Ici on est des prisonniers ambulants. Personne ne peut vous dire qu'il est content ici. Si on vous le dit, méfiez-vous parce que ce n'est pas vrai. Ici on est comme à l'école, c'est frustrant. On ne nous demande pas notre avis, comme si on n'était pas des personnes. Tout ce que je veux c'est récupérer mon autonomie. Ici on est lié des mains et des pieds, et on ne peut rien faire [...]. Tu arrives ici et tu as le sentiment d'être tombé encore plus bas... C'est une régression, tu es comme à l'école ou dans un internat, mais on est des adultes. » (Entretien avec Simone, demandeuse d'asile camerounaise, $1^{\text {er }}$ avril 2004)

L'organisation et la mise en œuvre de l'assistance publique offerte aux demandeurs d'asile dans le cadre du dispositif national d'accueil relève d'une forme de gouvernementalité ${ }^{39}$ qui repose sur des principes régulateurs de la

37. Decourcelle A., Julinet S., op. cit., p. 133.

38. La sensation de «soulagement » éprouvée par les demandeurs d'asile rencontrés ne peut se comprendre qu'au regard de leur parcours de circulation contrainte. Cette espèce de captivité bienveillante que constitue l'entrée en CADA doit être appréhendée au regard des trajectoires marquées par le mouvement et la perte de repères connus. Se trouver pendant quelques temps sous l'aile protectrice de l'Etat est souvent perçu comme source de "tranquillité », quoiqu'il s'agisse, tel que l'exprimait une jeune demandeuse d'asile somalienne, d' 'une tranquillité qui ne dure pas longtemps».

39. Foucault M., «Le sujet et le pouvoir », Dits et écrits, 1954-1988, tome IV, Paris, Gallimard, 2001. 
vie quotidienne. L'entrée au CADA implique l'assujettissement à des règles de conduite. Sans être un espace d'exception, le CADA est un espace de confinement où l'assistance ne va pas sans la perte d'autonomie des résidents vis-àvis des travailleurs sociaux. Or ces derniers ne sont pas de simples agents de contrôle exerçant le pouvoir, ils sont pris eux-mêmes dans des enjeux qui les mettent à mal.

\section{Expulser les déboutés}

«La préfecture est informée de la situation des résidents en CADA, elle est informée aussi des décisions de la Commission de recours et de l'OFPRA et donc la préfecture peut savoir qu'une personne hébergée en CADA est déboutée et le délai d'un mois passé, il peut $\mathrm{y}$ avoir des problèmes. Le directeur ne peut pas refuser l'accès des flics aux lieux communs mais on peut interdire l'accès aux chambres. Mais nous, on n'a jamais appelé la police pour qu'ils viennent chercher des gens... [...] Il y a des sorties qui se règlent par huissier, après trois refus, on contacte un huissier mais on n'a jamais fait venir la police [...]. Nous, pour l'instant on essaie de négocier avec la DDASS pour que les sorties ne se fassent pas trop mal [...] on ne sait pas ce qui va se passer dans le futur. »

(Entretien avec Bernard, directeur adjoint d'un CADA, 30 novembre 2007).

Le directeur adjoint d'un CADA décrivait ainsi la situation actuelle dans son établissement, marquée par la volonté d'éviter le délogement des déboutés au bout d'un mois et de négocier avec les autorités pour "gérer les sorties ». Dans un autre CADA les responsables tiennent, au contraire, selon leurs termes, à « respecter » les délais prévus par les documents officiels, « suivant au pied de la lettre » l'orientation entrepreneuriale du siège de l'association gestionnaire, ce qui ne va pas sans poser un certain nombre de difficultés pour les intervenants sociaux qui s'y opposent. Avant même le durcissement des dispositions, la rotation des entrées et des sorties de CADA était déjà visée :

[Commentant la réunion d'équipe :] « T'as vu ce matin Céline [une des responsables du CADA] dire que s'ils sont rejetés c'est plus facile. [Elle mime la scène] : “je n'ai aucun mal à virer les déboutés”. Tout est fait pour faire semblant... Ce n'est pas des personnes, que des chiffres, c'est pour ça qu'on a fait sortir les Ashi. Maintenant on préfère les déboutés, ça part plus vite. » (entretien avec Florence, intervenante dans un CADA, 17 février 2004).

"Faire sortir les déboutés », c'est-à-dire les expulser du centre de manière plus ou moins forcée, fait partie du « sale boulot» que les travailleurs sociaux 
doivent accomplir et qui, d'après la définition donnée par Everett Hughes ${ }^{40}$, correspond à des tâches qui sont physiquement dégoûtantes ou qui symbolisent quelque chose d'humiliant et de dégradant pour la personne. Ou encore, pour reprendre les termes de Christophe Desjours ${ }^{41}$, il s'agirait de l'enrôlement des braves gens dans «le mal » comme système de gestion, le «mal » conçu comme la tolérance et la participation à l'injustice et à la souffrance infligée à autrui.

«Ils vont être contraint de quitter les centres manu militari, je ne sais pas concrètement comment ça va se faire mais dans les textes c'est possible [...]. Et nous dans tout ça, je pense qu'on va basculer vers... on n'est plus dans l'humain. On va être des interlocuteurs des agences de voyages. [changeant la voix : ] T'es congolais, attend j'appelle l'ANAEM et on fixe une date pour le départ. Je vais venir habillé en gendarme. »

(entretien avec Estelle intervenante dans un CADA, 30 novembre 2007)

L'image du gendarme qu'évoque cette intervenante synthétise l'abandon des principes du travail social ainsi que la dégradation du métier. Non seulement les travailleurs sociaux sont soumis à une logique administrative, mais ils sont également des agents participant à une logique de contrôle. Bien entendu, il ne s'agit pas là d'une nouveauté qui apparaît avec le durcissement des mesures de sortie du CADA, mais il semblerait que ces dernières viennent rendre visible et inéluctable une réalité souvent refoulée par les intervenants. Si, pour ces professionnels, les «sorties » constituent un véritable dilemme, elles sont pour l'observateur des moments exemplaires où se cristallisent des tensions multiples entre les divers acteurs du confinement. Estelle nous donnait ainsi son avis sur ce que l'on appelle, au sein de l'équipe du CADA, une «sortie problématique » :

« [L'association] n'a pas de discours, et s'il y a un discours c'est de dire, "les déboutés, vous dégagez sinon on vous met un procès au cul”. Il s'est trouvé à un moment donné qu'on avait beaucoup, beaucoup de déboutés, pour plein de raisons, parce que les collègues n'ont pas fait leur travail au moment où il fallait, c'est pas un jugement de valeur, hein, je veux dire, il y a des... ils n'ont pas pu faire, ils n'ont pas pu, le président n'a pas pu, bon, pour X raisons on s'est trouvé avec plein de déboutés et parce qu'on avait la DDASS derrière, et parce que les lois ont changé et puis parce que... [l'association] aurait pu dire, comme ça a été le cas dans certains CADA, "nous, nous gardons les déboutés". Il $\mathrm{y}$ a des foyers qui ont dit ça. Quand [le président de l'association] a su qu'il hébergeait des clandestins et quand il a su qu'il pouvait être

40. Hughes E., Le Regard sociologique, Paris, Editions de l'EHESS, 1996.

41 . Desjours C., Souffrance en France. La banalisation de l'injustice sociale, Paris, Seuil, 1998. 
condamné [...] c'est de la lâcheté citoyenne. Et puis il y a un manque évident de militantisme. »

(Entretien avec Estelle, intervenante dans un CADA, 26 décembre 2005)

Cette "sortie problématique » a eu lieu bien avant le début de notre enquête dans ce CADA, cependant elle était toujours présente dans les esprits et apparaissait souvent au cours des conversations avec les professionnels. A l'époque, l'association avait envoyé des référés à des familles déboutées refusant de quitter le CADA et qui ont finalement été expulsées avant de faire appel à la police. Cette « sortie » rend compte des profonds désaccords entre les salariés du CADA - aussi bien les travailleurs sociaux que les responsables - et les autorités de l'association, comme l'illustre l'extrait ci-dessus. Le discours critique vis-à-vis du président de l'association est partagé par beaucoup d'intervenants du CADA qui agissent dans un esprit de "militance professionnelle 42 ». Cela n'est pas sans rappeler l'antinomie soulevée par Pierre Bourdieu entre la logique du travail social, "qui ne va pas sans un certain militantisme prophétique ou un bénévolat inspiré ", et celle de la bureaucratie, "de ses disciplines et de ses prudences " ${ }^{43}$. Les autorités du CADA essaient d'éviter l'intervention du siège de l'association - ce qui n'avait pas été le cas lors de cette "sortie problématique »- mais elles sont obligées d'informer le président des démarches d'expulsion et, bien entendu, en cas de procédures judiciaires lorsqu'il s'agit de ce qu'ils appellent des «problématiques lourdes». Le directeur adjoint du CADA me fait partager son expérience d'une "sortie problématique », qui fut d'ailleurs la première à laquelle il participa :

«Dans l'année où j’ai pris mes fonctions, ça faisait plusieurs années que les gens ne sortaient plus, ni déboutés ni réfugiés et on a eu donc des remarques de la DDASS et donc, quand j'ai pris mes fonctions, j'ai interpellé l'association pour savoir quelle était la position face aux déboutés. Il y avait un grand flou, personne ne disait rien donc... je savais qu'on n'était pas dans les règles demandées par la DDASS. Après coup, j'ai su qu'on était dans la même situation que les autres établissements CADA, c'était un problème pour tous, les gens ne sortaient plus [...]. Le directeur du CADA était en arrêt maladie, la DDASS voulait absolument qu'on fasse sortir ces gens et donc la problématique c'était... on avait réussi avec l'équipe à négocier que l'association prenne en charge une semaine de prise en charge en hôtel avec une semaine de repas, c'était un forcing de l'équipe vis-à-vis de l'association. Et en fait, il a fallu faire sortir au

42. Chibrac L., "L'héritage du travail social dans l'accueil des migrants", entretien réalisé par M. Poinsot avec L. Chibrac, Hommes et migrations, n'1261, mai-juin 2006, pp. 66-71, p. 71. 43 . Bourdieu P., «Une mission impossible », La Misère du monde, Paris, Seuil, 1993, p. 354. 
moment de Noël un jeune couple qui venait d'avoir un bébé, voilà et donc c'était pas très sympa, c'étaient des gens très sympathiques, des jeunes Kurdes qui nous ont fait confiance, la dame était en larmes et qu'on était obligé d'accompagner au fin fond du département, dans une zone industrielle...»

(Entretien avec Bernard, directeur-adjoint d'un CADA, 30 novembre 2007)

La jeune famille a quitté l'établissement de son plein gré, après maintes conversations avec les intervenants qui leur expliquaient qu' "il n'y avait pas d'autre option pour eux» que la « sortie » du CADA. Ce fragment rend compte de l'attachement émotionnel des intervenants à certains résidents et de la manière dont ils se mobilisent afin d'obtenir ne serait-ce que quelques jours de plus au foyer ou une semaine de prise en charge dans un hôtel après le CADA. Les travailleurs sociaux essaient de trouver les moyens pour que la « sortie » des déboutés se fasse, pour emprunter les termes d'un intervenant, "dans les meilleurs conditions possibles ", en essayant de leur ménager une sorte de transition avant de se retrouver dépourvus d'hébergement et d'assistance. Les référents tentent souvent d'autres voies de régularisation et mettent en œuvre des tactiques pour repousser le départ des déboutés, au moins, jusqu’à leur assurer une place pendant quelques semaines dans un hôtel du département :

«Si je peux leur donner des tickets pour le centre de loisirs ou négocier la gratuité pour $\mathrm{M}$ me $\mathrm{X}$, qui est partie il y a deux mois, la gratuité de la cantine pour la gamine... On essaie de contourner un peu comme on peut. C'est vrai que pour les déboutés on a toujours fonctionné comme ça, on essaie de leur filer tous les tickets qu'on peut, tickets de transport, tickets pour le centre de loisirs. Même quand ils partent on leur file des carnets, on essaie de les faire partir avec, on va dire, la situation administrative la moins pire. Aller au maximum de l'administratif avec le temps qu'on a. Tant qu'ils sont ici, ils ont une attestation d'hébergement, on entame toutes les démarches, on fait tout ce qu'on peut même s'ils partent demain [...]. Il y a comme une urgence à faire des choses, de l'orthophonie, des loisirs, du français, n’importe quoi, pour créer du réseau, pour créer des liens, on n'a pas beaucoup de temps, ça ne leur résout pas les problèmes mais on fait, on fait des choses. »

(Entretien avec Estelle, intervenante dans un CADA, 30 novembre 2007)

C'est grâce à l'initiative des fonctionnaires les moins « emprisonnés » dans leur fonction, et qui dispose toujours d'une certaine marge de manœuvre, bien que de plus en plus restreinte, que l'on arrive à «faire du social ». Le directeur-adjoint trouve cependant un moyen de justifier l'expulsion des déboutés, probablement pour rendre « la sortie » moralement acceptable, en arguant que 
cela constitue une façon de remettre les résidents "dans les rails de l'aide sociale », le refus de sortir du CADA faisant obstacle à cette dernière :

« Mais en même temps c'était bien parce que ça les remettait dans les rails de l'aide sociale parce que, quand les gens refusent de quitter le CADA, ça les met en défaut et nous on ne peut pas faire grande chose. »

(Entretien avec Bernard, directeur-adjoint dans un CADA, 30 novembre 2007)

Les «sorties » peuvent aussi rendre compte des dissensions entre les responsables du CADA. Dans nos conversations, Bernard critique de manière voilée l'attitude et les décisions prises en dernière instance par le directeur de l'établissement qui, d'après lui, aurait dû non seulement émettre son avis mais, surtout, négocier auprès du siège de l'association afin de prolonger le séjour des déboutés.

Si dans l'un des CADA où nous avons mené notre terrain les «sorties » se font de manière systématique une fois épuisé le délai officiel, dans l'autre CADA, les autorités essayaient de négocier avec la DDASS le prolongement du séjour des déboutés. Or, depuis la fin 2007, et plus clairement encore en 2008, d'après les conversations que nous avons pu avoir avec l'équipe du CADA en d'avril, plus aucun délai supplémentaire n'est accordé, plus aucune marge de manœuvre de la part des responsables d'établissement pour héberger les déboutés au-delà d'un mois n'est permise.

\section{Enfermés dans un dilemme}

Dans son travail sur l'accueil étatisé des demandeurs d'asile, Jérôme Valluy ${ }^{44}$ argumente que, du fait de l'enrôlement d'acteurs associatifs dans les dispositifs de gestion des CADA, les mobilisations sociales en faveur des demandeurs d'asile sont subordonnées aux finalités de l'Etat. Il montre bien qu'il y a eu une adaptation progressive des CADA aux idéologies étatiques qui orientent aujourd'hui l'action publique en matière de migrations et d'asile en France. L'étude ethnographique menée sur le quotidien des travailleurs sociaux dans les CADA permet, en revanche, de nuancer cette hypothèse pour ce qui est de l'intériorisation des perceptions étatiques de la part des intervenants qui côtoient les demandeurs d'asile au jour le jour. Les travailleurs sociaux des CADA tendent, selon Jérôme Valluy, à raisonner dans les mêmes termes que les fonctionnaires étatiques des administrations et des tribunaux. Or, les extraits d'entretiens avec les intervenants des CADA présentés plus

44. Valluy J., «L'accueil étatisé des demandeurs d'asile : de l'enrôlement dans les politiques publiques à l'affaiblissement des mobilisations de soutien aux exilés ", op. cit ; Valluy J., Sociologie politique de l'accueil et du rejet des exilés, op. cit. 
haut rendent compte du dilemme que constitue pour eux le fait d'être agents d'une politique qu'ils considèrent comme éloignée des principes de leur travail. Il s'agit d'un véritable dilemme - et c'est aussi le terme employé par un de nos interlocuteurs - dans le sens où ils doivent choisir entre les deux termes contradictoires et également insatisfaisants d'une alternative : les "faire sortir » implique de s'assumer comme un agent pris dans une logique qu'ils contestent; se refuser à accomplir ce travail, c'est-à-dire refuser de procéder à l'expulsion en arguant par exemple que la famille déboutée en question a un bébé ou qu'elle n'arrive pas à trouver un hôtel, mettrait en cause leur aptitude à exercer leur travail, ce qui risquerait de leur faire perdre leur emploi. Quoi qu'ils fassent, rien ne permettra aux résidents de rester au CADA.

La question qui se pose aujourd'hui, au-delà de l'association entre assistance et contrôle, est celle du rôle du travail social dans l'éloignement des étrangers du territoire national et communautaire. Que ce soit en fournissant aux préfets des données sur les résidents ou en incitant les déboutés à retourner dans leurs pays - tel que le laissait paraître un fragment d'entretien cité plus haut -, le travail social en CADA devient désormais le complément ou, pour emprunter un autre terme, le «supplétif de la préfecture 45 ».

Commentant la situation de la famille albanaise décrite dans Libération (et qui ouvrecet article), un intervenant observait, résigné, « on a beau faire mais il n'y a que l'expulsion à l'horizon ». " Faire du social » lorsque l'horizon des pratiques assistantielles n'est autre que l'expulsion des étrangers semble, sinon une mission impossible, au moins un véritable défi.

45. Christophe Daadouch montre comment, avec les dernières lois en matière d'attestation d'accueil, de regroupement familial, de processus de régularisation et concernant les mineurs étrangers isolés, le travail social devient un «supplétif de la préfecture » pour ce qui est des vérifications de logement, des avis d'intégration, etc. Daadouch C., «Vers une logique de contrôle ?", Plein droit, nº70, octobre 2006, pp. 14-18. 